\title{
Detection of Inducible Nitric Oxide Synthase- and Nitrotyrosine-Positive Cells in the Lesions of Pericarditis Induced by Porcine Enterovirus Serotype 3 Infection
}

\author{
Taekyun SHIN ${ }^{1)}$, Sungsoo CHO ${ }^{2)}$ and Chasoo LEE ${ }^{3)}$ \\ ${ }^{1)}$ Department of Veterinary Medicine, Institute for Life Science, Cheju National University, Jeju city 690-756, ${ }^{2)}$ ChoongAng Animal \\ Disease Laboratory and ${ }^{3)}$ College of Veterinary Medicine, Kyungpook National University, Taegu, Republic of Korea
}

(Received 7 March 2001/Accepted 14 May 2001)

ABSTRACT. The expression of inducible nitric oxide synthase (iNOS), an enzyme that produces nitric oxide, was examined in the hearts of pigs infected with porcine enterovirus serotype 3 (PEV3). Piglets orally infected with PEV3 developed tremors and paralysis 3-7 days post-infection. Affected animals had pericarditis and myocarditis. There were fibrin and inflammatory cell infiltrates (macrophages and neutrophils) in the pericardial sac and myocardium. Immunohistochemically, the majority of inflammatory cells in the pericardial sac were positive for iNOS and nitrotyrosine, an end product of nitric oxide. These results suggest that iNOS is upregulated in the pericardial lesion, and that increased nitric oxide production plays an important role in the development of PEV3-induced pericarditis and myocarditis.

KEY WORDS: nitric oxide synthase (NOS), pericarditis, porcine enterovirus 3.

J. Vet. Med.Sci. 63(9): 1017-1019, 2001

Porcine enterovirus serotype 3 (PEV3) is a pathogen causing pericarditis and myocarditis in pigs, its natural host [2]. It is believed that the disease process in PEV3 infection is initiated by viremia, which is followed by the infiltration of inflammatory cells into various organs, including the brain and heart [1]. We have classified the serotypes of PEV isolated from the feces, milk, and brains of pigs in Korea [9], and have also examined the pathogenicity of PEV3 in colostrum-deprived pigs [7, 8]. However, the exact mechanism underlying the cardiac injury in PEV3 infection is not fully understood.

Nitric oxide is a readily diffusible nonpolar gas that is formed in a variety of cell types; it influences a number of physiological and pathological conditions [5]. The enzyme responsible for nitric oxide production exists in two forms: (1) constitutive nitric oxide synthase (cNOS), which is readily activated by agonists that elevate intracellular free $\mathrm{Ca}^{2+}$, and (2) inducible NOS (iNOS), which is induced following several hours of immunological stimulation. The biological reactions controlled by nitric oxide are diverse, and include immune cell-mediated cytotoxicity, regulation of vascular tone and relaxation, inhibition of platelet aggregation, and neural transmission [5].

Accumulating evidence suggests that nitric oxide may be involved in the pathogenesis of Coxsackie B3 virus myocarditis $[4,6]$, similar to its involvement in autoimmune myocarditis in rat models [10]. The identification of specific mediators, such as nitric oxide in PEV3 infections, may help in the study of disease pathogenesis. In this study, we investigated the expression of iNOS and nitrotyrosine, which are a potent nitric oxide-producing enzyme and an end product of nitric oxide, respectively, in pericarditis and myocarditis lesions in piglets infected with PEV3.

Colostrum-deprived newborn piglets $(n=6)$ were orally administered $1 \mathrm{~m} l$ of tissue culture suspension containing PEV3, within $48 \mathrm{hr}$ of birth. The inoculum contained $10^{7.5}$
$\mathrm{TCID}_{50}$ (tissue culture infectious doses) of virus per $\mathrm{ml}$. Two control piglets were given the same volume of tissue culture suspension. Each group was maintained in a separate isolator. The PEV3 used in this study was originally isolated from the milk of a pig with neurological signs [7, 8].

The animals were anesthetized with pentobarbital sodium, and sacrificed on days 3, 5, and 7 post-infection ( $\mathrm{n}=2$ each). Various organs, including the brain, lungs, and heart, were removed and fixed in $10 \%$ buffered formalin. Paraffin sections of the hearts were cut and stained with hematoxylin and eosin (HE).

Immunohistochemical staining of iNOS and nitrotyrosine was carried out using an Avidin-Biotin Complex Kit (Vector, Burlingame, CA, U.S.A.) as instructed by the manufacturer, and as previously described [10]. The antisera used in this study were rabbit anti-iNOS polyclonal antiserum (1:1000) (Transduction Laboratory, Lexington, CA, U.S.A.) and rabbit anti-nitrotyrosine polyclonal antiserum (1:200) (Upstate Biotechnology Inc., NY, U.S.A.).

Five- $\mu \mathrm{m}$ sections of heart were deparaffinized, and treated with $0.3 \%$ hydrogen peroxide in methanol for $30 \mathrm{~min}$ to block endogenous peroxidase. After three washes, the sections were exposed to normal goat serum, and then incubated with each primary antiserum diluted in PBS for $1 \mathrm{hr}$ at room temperature. After washing, the sections were sequentially treated with biotinylated goat anti-rabbit immunoglobulin and avidin-biotin peroxidase complex, developed with diaminobenzidine-hydrogen peroxide solution $(0.001 \%$ 3,3'-diaminbenzidine and $0.01 \%$ hydrogen peroxide in $0.05 \mathrm{M}$ Tris buffer), and counterstained with hematoxylin.

Heart tissues were minced, and fixed in $2.5 \%$ glutaraldehyde in cacodylate buffer, post-fixed in $1 \%$ osmium tetroxide, dehydrated through graded dilutions of alcohol, and embedded in Epon 812. Ultrathin sections were stained with uranyl acetate and lead citrate, and examined with a 


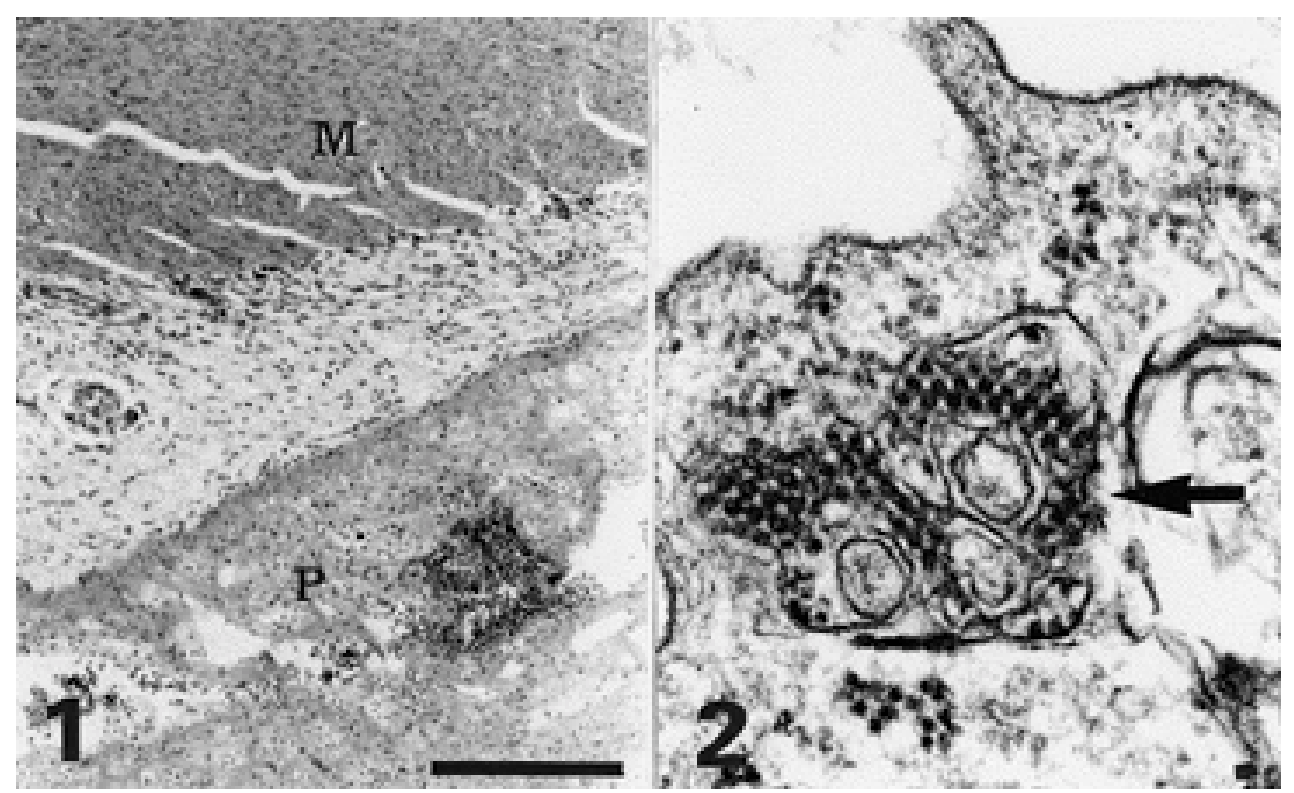

Fig. 1. Histology of a PEV3-infected heart at day 7 post-infection. The inflammatory reaction extended from the pericardium $(\mathrm{P})$ to the epicardium and further into the myocardium $(\mathrm{M})$. Note the infiltration of inflammatory cells into the pericardial sac. HE stain. Bar $=100 \mu \mathrm{m}$.

Fig. 2. Ultrastructure of a vascular endothelial cell in a PEV3-infected heart at day 7 post-infection. Crystalline arrays of virus-like particles are present (arrow). Bar=100 nm.

JEM 1200 EX electron microscope (JEOL, Tokyo, Japan).

The clinical course of PEV3 infection in colostrumdeprived pigs has been well documented in previous reports [7, 8]. PEV3-infected pigs develop tremor and paralysis, beginning on days 3 to 7 post-infection. In this study, we focused on changes in the heart, with special emphasis on iNOS expression. At necropsy, pericardial thickening and fibrinous coagulative tissue were evident at days 5 and 7 post-infection in two infected animals, whereas control pigs showed no pathologic changes in the heart.

Histopathologically, the coagulative tissue in the pericardial sac contained clusters of inflammatory cells (macrophages and neutrophils) and fibrin (Fig. 1). An inflammatory reaction with fibrous proliferation was present in the pericardium and epicardium, and extended into the superior myocardium. Individual myocardial fibers in the lesions were necrotic or calcified. Ultrastructurally, we observed dilation of the rough endoplasmic reticulum, and crystalline arrays of virus-like particles in vascular endothelial cells, at day 7 post-infection (Fig. 2).

Immunohistochemically, iNOS-positive cells (Fig. 3A), probably macrophages, were present in the fibrinous clusters in the pericardial sac and in the interstitial tissue of the myocardium adjacent to the epicardium. There were relatively few iNOS-positive cells in the deeper myocardium. Many iNOS-positive cells were also positive for nitrotyrosine (Fig. 3B) in mirror-image sections, which suggests the generation of nitric oxide.

This is the first report of the presence of iNOS- and nitrotyrosine-positive inflammatory cells in the pericardial sac during PEV3 infection. Nitric oxide produced by iNOS is proposed to be an important effector molecule in the pathogenesis of viral myocarditis [3, 4, 11], and in a variety of animal models, including autoimmune pericarditis and myocarditis [10]. The functional role of nitric oxide in viral myocarditis, however, remains controversial. Nitric oxide is known to be involved in tissue injury during viral myocarditis [6], and is inversely related to host defenses because it inhibits viral replication $[3,11]$. However, there is general agreement that nitric oxide mediates tissue injury in the early stage of inflammation.

In this study, we clarified the involvement of nitric oxide in the pathogenesis of PEV3-induced acute pericarditis and myocarditis in pigs. In the lesions, nitric oxide produced in inflammatory cells (probably macrophages) may mediate vascular leakage and tissue injury, and exacerbate inflammation. We postulate that the expression of iNOS, which generates nitric oxide, is involved in disease initiation at the early viremic stage of PEV3 infection. PEV3 may infect vascular endothelial cells and increase vascular permeability in the pericardial sac, and infiltrated macrophages may generate nitric oxide in this experimental model. Nitric oxide produced at such high levels may further increase vascular permeability, and may be toxic to the adjacent epicardium and myocardium.

ACKNOWLEDGMENT. The authors thank Professor Kunitoshi Mitsumori, Laboratory of Veterinary Pathology, Tokyo University of Agriculture and Technology, Tokyo, Japan for critical comments on this thesis (T. Shin). 


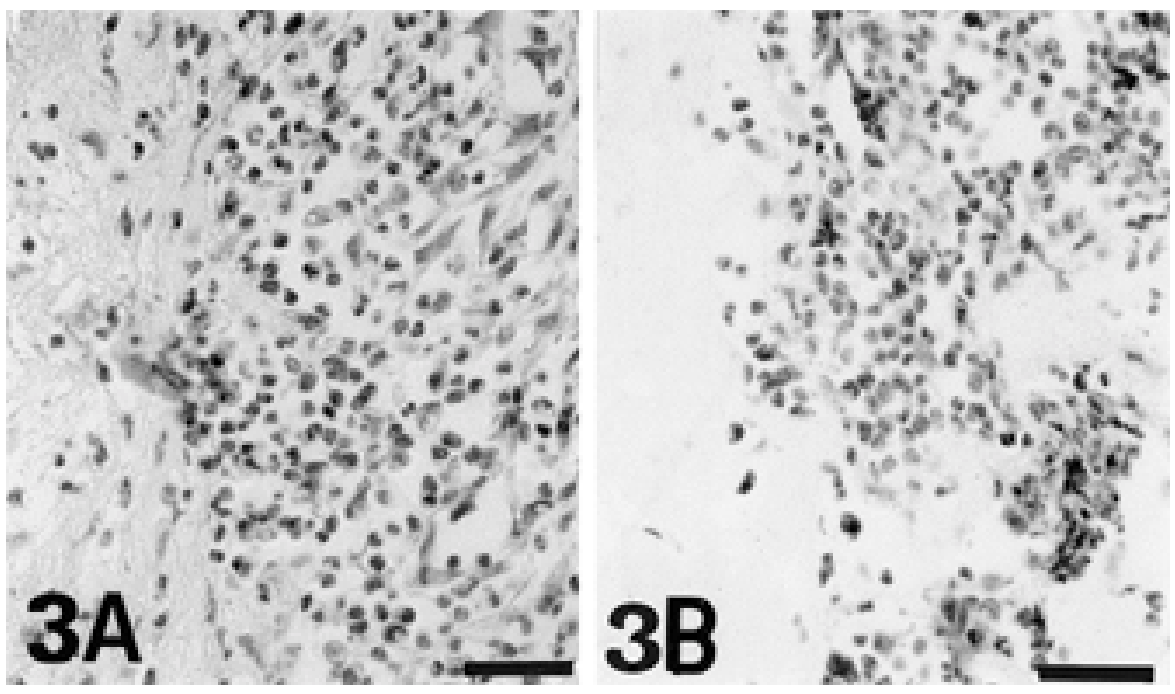

Fig. 3. Immunostaining for iNOS and nitrotyrosine in a PEV3-infected heart at day 7 post-infection. Some of the inflammatory cells, probably macrophages, in the pericardial sac were positive for iNOS (A) and nitrotyrosine (B). Counterstained with hematoxylin. Bar $=30 \mu \mathrm{m}$.

\section{REFERENCES}

1. Baba, S. P., Bohl, E. H. and Meyer, R. C. 1966. Cornell Vet. 56: $385-394$.

2. Long, J. F., Kasza, L. and Koestner, A. 1969. J. Infect. Dis. 120: $245-249$.

3. Lowenstein, C. J., Hill, S. L., Lafond-Walker, A., Wu, J., Allen, G., Landavere, M., Rose, N. R. and Herskowitz, A. 1966. J. Clin. Invest. 97: 1837-1843.

4. Mikami, S., Kawashima, S., Kanazawa, K., Hirata, K., Katayama, Y., Hotta, H., Hayashi, Y., Ito, H. and Yokoyama, M. 1996. Biochem. Biophys. Res. Commun. 220: 983-989.

5. Moncada, S., Palmer, R. M. and Higgs, E. A. 1991. Pharmacol. Rev. 43: 109-142.
6. Robinson, N. M., Zhang, H. Y., Bevan, A. L., De Belder, A. J., Moncada, S., Martin, J. F. and Archard, L. C. 1999. Eur. J. Clin. Invest. 9: 700-707.

7. Shin, T. and Lee, C. 1985. Korean J. Vet. Res. 25: 103-112.

8. Shin, T. and Lee, C. 1988. Korean J. Vet. Res. 28: 137-143.

9. Shin, T., Lee, C., Kwon, H. and Knowles, N. J. 1987. Korean J. Vet. Res. 27: 223-226.

10. Shin, T., Tanuma, N., Kim, S., Jin, J., Moon, C., Kim, K., Kohyama, K., Matsumoto, Y. and Hyun, B. 1998. J. Neuroimmunol. 92: 133-138.

11. Zaragoza, C., Ocampo, C., Saura, M., Leppo, M., Wei, X. Q., Quick, R., Moncada, S., Liew, F. Y. and Lowenstein, C. J. 1988. Proc. Natl. Acad. Sci. U.S.A. 95: 2469-2474. 